\title{
ON MOORE-YAMASAKI-KHARAZISHVILI TYPE MEASURES AND THE INFINITE POWERS OF BOREL DIFFUSED PROBABILITY MEASURES ON R
}

\author{
M.KINTSURASHVILI, T.KIRIA AND G.PANTSULAIA
}

\begin{abstract}
The paper contains a brief description of Yamasaki's remarkable investigation (1980) of the relationship between Moore-Yamasaki-Kharazishvili type measures and infinite powers of Borel diffused probability measures on $\mathbf{R}$. More precisely, we give Yamasaki's proof that no infinite power of the Borel probability measure with a strictly positive density function on $R$ has an equivalent Moore-Yamasaki-Kharazishvili type measure. A certain modification of Yamasaki's example is used for the construction of such a Moore-YamasakiKharazishvili type measure that is equivalent to the product of a certain infinite family of Borel probability measures with a strictly positive density function on $R$. By virtue of the properties of equidistributed sequences on the real axis, it is demonstrated that an arbitrary family of infinite powers of Borel diffused probability measures with strictly positive density functions on $R$ is strongly separated and, accordingly, has an infinite-sample well-founded estimator of the unknown distribution function. This extends the main result established in [ Zerakidze Z., Pantsulaia G., Saatashvili G. On the separation problem for a family of Borel and Baire $G$-powers of shift-measures on $\mathbb{R} / /$ Ukrainian Math. J. -2013.- 65 (4).- P. 470-485 ].
\end{abstract}

\section{INTRODUCTION}

Let $\mu$ and $\nu$ be non-trivial $\sigma$-finite measures on a measurable space $(X, M)$. The measures $\mu$ and $\nu$ are called orthogonal if there is a measurable set $E \in M$ such that $\mu(E)=0$ and $\nu(X \backslash E)=0$. The measures $\mu$ and $\nu$ are called equivalent if and only if the following condition

$$
(\forall E)(E \in M \rightarrow(\mu(E)=0 \Longleftrightarrow \nu(E)=0))
$$

is satisfied.

It is well known that the following facts hold true in an $n$-dimensional Euclidean vector space $\mathbf{R}^{n}(n \in N)$ :

Fact 1.1 Let $\mu$ be a probability Borel measure on $\mathbf{R}$ with a strictly positive continuous distribution function and $\lambda_{n}$ be a Lebesgue measure defined on the $n$ dimensional topological vector space $\mathbf{R}^{n}$. Then the measures $\mu^{n}$ and $\lambda_{n}$ are equivalent.

1991 Mathematics Subject Classification. 60B15, 28C15 .

Key words and phrases. Moore-Yamasaki-Kharazishvili type measure, product measure, equidistributed sequence of real numbers.

The third author partially is supported by Shota Rustaveli National Science Foundation's Grant no 31/25. 
Fact 1.2 Let $\left(\mu_{k}\right)_{1 \leq k \leq n}$ be a family of Borel probability measures on $\mathbf{R}$ with strictly positive continuous distribution functions and $\lambda_{n}$ be a Lebesgue measure defined on the n-dimensional topological vector space $\mathbf{R}^{n}$. Then the measures $\prod_{k=1}^{n} \mu_{k}$ and $\lambda_{n}$ are equivalent.

Fact 1.3 Let $\mu_{1}$ and $\mu_{2}$ be Borel probability measures on $\mathbf{R}$ with strictly positive continuous distribution functions. Then the measures $\mu_{1}^{n}$ and $\mu_{2}^{n}$ are equivalent.

Fact 1.4 Let $\left(\mu_{k}\right)_{1 \leq k \leq n}$ be a family of Borel probability measures on $\mathbf{R}$ with strictly positive continuous distribution functions. Then the measures $\mu_{k}^{n}$ and $\mu_{l}^{n}$ are equivalent for each $1 \leq k \leq l \leq n$.

The proof of the above mentioned facts employs the following simple lemma which is well known in the literature.

Lemma 1.1 Let $\mu_{k}$ and $\nu_{k}$ be equivalent non-trivial $\sigma$-finite Borel measures on the measurable space $\left(X_{k}, M_{k}\right)$ for $1 \leq k \leq n$. Then the measures $\prod_{k=1}^{n} \mu_{k}$ and $\prod_{k=1}^{n} \nu_{k}$ are equivalent.

Proof. Note that for proving Lemma 1.1, it suffices to prove that if $\mu_{k}$ is absolutely continuous with respect to $\nu_{k}(k=1,2)$, then so is $\prod_{k=1}^{2} \mu_{k}$ with respect to $\prod_{k=1}^{2} \nu_{k}$.

Assume that $E \in M_{1} \times M_{2}$ such that $\mu_{1} \times \mu_{2}(E)=0$. We have to show that $\nu_{1} \times \nu_{2}(E)=0$.

By the Fubini theorem we have

$$
0=\mu_{1} \times \mu_{2}(E)=\int_{X_{1}} \mu_{2}\left(E \cap\left(\{x\} \times X_{2}\right)\right) d \mu_{1}(x) .
$$

This means that

$$
\mu_{1}\left(\left\{x: \mu_{2}\left(E \cap\left(\{x\} \times X_{2}\right)\right)>0\right\}\right)=0
$$

or, equivalently,

$$
\mu_{1}\left(X_{1} \backslash\left\{x: \mu_{2}\left(E \cap\left(\{x\} \times X_{2}\right)\right)=0\right\}\right)=0 .
$$

Since $\nu_{1} \ll \mu_{1}$, we have

$$
\left\{x: \mu_{2}\left(E \cap\left(\{x\} \times X_{2}\right)\right)=0\right\} \subseteq\left\{x: \nu_{2}\left(E \cap\left(\{x\} \times X_{2}\right)\right)=0\right\} .
$$

Since $\nu_{1} \ll \mu_{1}$ and

$$
\mu_{1}\left(X_{1} \backslash\left\{x: \nu_{2}\left(E \cap\left(\{x\} \times X_{2}\right)\right)=0\right\}\right)=0,
$$

we have

$$
\nu_{1}\left(X_{1} \backslash\left\{x: \nu_{2}\left(E \cap\left(\{x\} \times X_{2}\right)\right)=0\right\}\right)=\nu_{1}\left(\left\{x: \nu_{2}\left(E \cap\left(\{x\} \times X_{2}\right)\right)>0\right\}\right)=0 .
$$

Finally, we get

$$
\begin{gathered}
\nu_{1} \times \nu_{2}(E)=\int_{X_{1}} \nu_{2}\left(E \cap\left(\{x\} \times X_{2}\right)\right) d \nu_{1}(x)= \\
\int_{\left\{x: \nu_{2}\left(E \cap\left(\{x\} \times X_{2}\right)\right)>0\right\}} \nu_{2}\left(E \cap\left(\{x\} \times X_{2}\right)\right) d \nu_{1}(x)+ \\
\int_{\left\{x: \nu_{2}\left(E \cap\left(\{x\} \times X_{2}\right)\right)=0\right\}} \nu_{2}\left(E \cap\left(\{x\} \times X_{2}\right)\right) d \nu_{1}(x)=0 .
\end{gathered}
$$


In order to obtain the infinite-dimensional versions of Facts 1.1-1.2, we must know what measures in infinite-dimensional topological vector spaces can be taken as partial analogs of the Lebesgue measure in $\mathbf{R}^{n}(n \in N)$. In this direction the results of I. Girsanov and B. Mityagyn [5] and Sudakov [16] on the nonexistence of nontrivial translation-invariant $\sigma$-finite Borel measures in infinite-dimensional topological vector spaces are important. These authors assert that the properties of $\sigma$-finiteness and of translation-invariance are not consistent. Hence one can weaken the property of translation-invariance for analogs of the Lebesgue measure and construct nontrivial $\sigma$-finite Borel measures which are invariant under everywhere dense linear manifolds. We wish to make a special note that Moore [12, Yamasaki [19] and Kharazishvili [9] give the constructions of such measures in an infinite-dimensional Polish topological vector space $\mathbb{R}^{\mathbb{N}}$ of all real-valued sequences equipped with product topology, which are invariant under the group $R^{(N)}$ of all eventually zero real-valued sequences. Such measures can be called MooreYamasaki-Kharazishvili type measures in $\mathbb{R}^{\mathbb{N}}$. Using Kharazishvili's approach [9], it is proved in [3] that every infinite-dimensional Polish linear space admits a $\sigma$-finite non-trivial Borel measure that is translation invariant with respect to a dense linear subspace. This extends a recent result of Gill, Pantsulaia and Zachary 4 on the existence of such measures in Banach spaces with Schauder bases.

In this paper, we focus on the question whether Facts 1.1-1.2 admit infinitedimensional generalizations in terms of Moore-Yamasaki-Kharazishvili type measures in $\mathbb{R}^{\mathbb{N}}$. To this end, our consideration will involve the following problems.

Problem 1.1 Let $\mu$ be a probability Borel measure on $R$ with a strictly positive continuous distribution function and $\lambda$ be a Moore-Yamasaki-Kharazishvili type measure in $\mathbb{R}^{\mathbb{N}}$. Are the measures $\mu^{N}$ and $\lambda$ equivalent?

Problem 1.2 Let $\left(\mu_{k}\right)_{k \in N}$ be a family of Borel probability measures on $R$ with strictly positive continuous distribution functions and $\lambda$ be a Moore-YamasakiKharazishvili type measure in $\mathbb{R}^{\mathbb{N}}$. Are the measures $\prod_{k \in N} \mu_{k}$ and $\lambda$ equivalent?

Concerning Facts 1.3-1.4, it is natural to consider the following problems.

Problem 1.3 Let $\mu_{1}$ and $\mu_{2}$ be Borel probability measures on $R$ with strictly positive continuous distribution functions. Are the measures $\mu_{1}^{N}$ and $\mu_{2}^{N}$ equivalent?

Problem 1.4 Let $\left(\mu_{i}\right)_{i \in I}$ be a family of all Borel probability measures on $R$ with strictly positive continuous distribution functions. Setting $S\left(R^{N}\right):=\cap_{i \in I} \operatorname{dom}\left(\overline{\mu_{i}^{N}}\right)$, where $\overline{\mu_{i}^{N}}$ denotes a usual completion of the measure $\mu_{i}^{N}(i \in I)$, does there exist a partition $\left(D_{i}\right)_{i \in I}$ of $R^{N}$ into elements of the $\sigma$-algebra $S\left(R^{N}\right)$ such that $\overline{\mu_{i}^{N}}\left(D_{i}\right)=1$ for each $i \in I$ ?

Problems 1.3-1.4 are not new and have been investigated by many authors in more general formulations. In this direction, we should specially mention the result of S. Kakutani [8] (see Theorem 4.3) stating that if one has equivalent probability measures $\mu_{i}$ and $\nu_{i}$ on the $\sigma$-algebra $\mathcal{L}_{i}$ of subsets of a set $\Omega_{i}, i=1,2, \cdots$ and if $\mu$ and $\nu$ denote respectively the infinite product measures $\prod_{i \in N} \mu_{i}$ and $\prod_{i \in N} \nu_{i}$ on the infinite product $\sigma$-algebra generated on the infinite product set $\Omega$, then $\mu$ and $\nu$ are either equivalent or orthogonal. Similar dichotomies have revealed themselves in the study of Gaussian stochastic processes. C. Cameron and W.E. Martin [1] that if one considers the measures induced on a path space by a Wiener process on the unit interval, then, if the variances of corresponding processes are 
different, the measures are orthogonal. Results of this kind were generalized by many authors(cf. [2], 6] and others). A.M. Vershik [17] proved that a group of all admissible translations(in the sense of quasiinvariance) of an arbitrary Gaussian measure in an infinite-dimensional separable Hilbert space is a linear manifold.

For study of the general problem of equivalence and singularity of two product measures was carried out by various authors using different approaches, among which are the strong law of large numbers, the properties of the Hellinger integral [7, the zero-one laws [11] and so on. In this paper, we propose a new approach for the solution of Problems 1.3-1.4, which uses the properties of uniformly distributed sequences [10].

In Sections 2-3, we give solutions of Problems 1.1-1.2 which are due to Yamasaki [19. In Section 4, we give solutions of Problems 1.3-1.4.

\section{Negative Solution of the Problem 1.1}

A negative solution of Problem 1.1 is contained in the following

Fact 2.1([19], Proposition 2.1, p. 696) Let $f(x)$ be a measurable function on $R^{1}$ which satisfies $f(x)>0$ and $\int_{-\infty}^{+\infty} f(x) d x=1$. Let $\mu$ be the stationary product measure of $f$ (i.e. $d \mu=\prod_{i=1}^{\infty} f\left(x_{i}\right) d x_{i}$ ) and $\mathbf{R}^{(\mathbf{N})}$ be a linear vector space of all eventually zero real-valued sequences. Then $\mu$ is $\mathbf{R}^{(\mathbf{N})}$-quasi-invariant but $\mu$ has no equivalent Moore-Yamasaki-Kharazishvili type measure.

Proof. As proved in [14, the stationary product measure $\mu$ is $\mathbf{R}^{(\mathbf{N})}$-ergodic. Let $\sum$ be the permutation group on the set of all natural numbers $N=\{1,2, \ldots\} . \sum$ can be regarded as a transformation group on $\mathbf{R}^{\mathbf{N}}$, and $\mu$ is $\sum$-invariant. Let $\sum_{0}$ be the subgroup of $\sum$ generated by all transpositions (of two elements of $N$ ). $\sum_{0}$ consists of such a permutation $\sigma \in \sum$ that satisfies $\sigma(i)=i$ except finite numbers of $i \in N$. As shown in [14, the measure $\mu$ is $\sum_{0}$-ergodic.

Now, we shall derive a contradiction assuming that $\mu$ has an equivalent $\mathbf{R}^{(\mathbf{N})}$ invariant $\sigma$-finite measure $\nu$. Since $\mu \approx \nu$, where $\mu$ is $\sum_{0}$-invariant and $\sum_{0}$ - ergodic, and $\nu$ is $\sum_{0}$-invariant, then we have $\mu=c \nu$ for some constant $c>0$. Thus, the

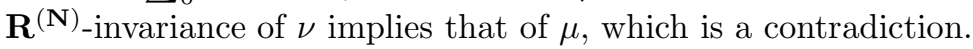

Therefore it suffices to prove that $\nu$ is $\sum_{0}$-invariant, namely for each $\sigma \in \sum_{0}$, $\tau_{\sigma} \nu=\nu$, where

$$
\tau_{\sigma} \nu(B)=\nu\left(\sigma^{-1}(B)\right)
$$

for each $B \in \mathbb{B}\left(R^{N}\right)$. Since $\tau_{\sigma} \mu=\mu$, we have $\tau_{\sigma} \nu \approx \nu$. On the other hand, $\nu$ is $\mathbf{R}^{(\mathbf{N})}$-ergodic because $\mu$ is such. Therefore if $\tau_{\sigma} \nu$ is $\mathbf{R}^{(\mathbf{N})}$-invariant, then we have $\tau_{\sigma} \nu=c_{\sigma} \nu$ for some constant $c_{\sigma}>0$. In particular for a transposition $\sigma$, $\sigma^{2}=l$ implies $c_{\sigma}^{2}=l$, hence $c_{\sigma}=1$. This means that $\nu$ is invariant under any transposition. Since $\sum_{0}$ is generated by the set of all transpositions, we have proved the $\sum_{0}$-invariance of $\nu$.

To complete the proof of Fact 2.1, it remains only to prove that $\tau_{\sigma} \nu$ is $\mathbf{R}^{(\mathbf{N})}$ invariant. Since $\nu$ is $\mathbf{R}^{(\mathbf{N})}$-invariant, we have $\tau_{x} \nu=\nu$ for any $x \in \mathbf{R}^{(\mathbf{N})}$. Therefore

$$
(\forall x)\left(x \in \mathbf{R}^{(\mathbf{N})} \rightarrow \tau_{\sigma} \tau_{x} \nu=\tau_{\sigma} \nu\right) .
$$

However, we can easily show $\tau_{\sigma} \tau_{x} \nu=\tau_{\sigma x} \tau_{\sigma} \nu$, so (2.2) implies that $\tau_{\sigma}$ is $\sigma\left(\mathbf{R}^{(\mathbf{N})}\right)$ invariant. Since $\sigma$ maps $\mathbf{R}^{(\mathbf{N})}$ onto $\mathbf{R}^{(\mathbf{N})}$, namely $\sigma\left(\mathbf{R}^{(\mathbf{N})}\right)=\mathbf{R}^{(\mathbf{N})}$ we have proved the $\mathbf{R}^{(\mathbf{N})}$-invariance of $\tau_{\sigma} \nu$. 


\section{Particular solution of Problem 1.2}

Remark 3.1. If in the formulation of Problem 1.2 we have that $\mu_{k}=\mu_{n}$ for each $k, n \in N$, then, following Fact 2.1, the answer to the question posed in Problem 2.1 is no.

Example 3.1.(13, Section 1, p. 354 ). Let $\mathbf{R}^{N}$ be the topological vector space of all real-valued sequences equipped with the Tychonoff topology. Let us denote by $B\left(\mathbf{R}^{N}\right)$ the $\sigma$-algebra of all Borel subsets in $\mathbf{R}^{N}$.

Let $\left(a_{i}\right)_{i \in N}$ and $\left(b_{i}\right)_{i \in N}$ be sequences of real numbers such that

$$
(\forall i)\left(i \in N \rightarrow a_{i}<b_{i}\right)
$$

We put

$$
A_{n}=\mathbf{R}_{0} \times \cdots \times \mathbf{R}_{n} \times\left(\prod_{i>n} \Delta_{i}\right)
$$

for $n \in N$, where

$$
(\forall i)\left(i \in N \rightarrow \mathbf{R}_{i}=\mathbf{R} \& \Delta_{i}=\left[a_{i} ; b_{i}[) .\right.\right.
$$

We put also

$$
\Delta=\prod_{i \in N} \Delta_{i}
$$

For an arbitrary natural number $i \in N$, consider the Lebesgue measure $\mu_{i}$ defined on the space $\mathbf{R}_{i}$ and satisfying the condition $\mu_{i}\left(\Delta_{i}\right)=1$. Let us denote by $\lambda_{i}$ the normed Lebesgue measure defined on the interval $\Delta_{i}$.

For an arbitrary $n \in N$, let us denote by $\nu_{n}$ the measure defined by

$$
\nu_{n}=\prod_{1 \leq i \leq n} \mu_{i} \times \prod_{i>n} \lambda_{i}
$$

and by $\bar{\nu}_{n}$ the Borel measure in the space $\mathbf{R}^{N}$ defined by

$$
(\forall X)\left(X \in B\left(\mathbf{R}^{N}\right) \rightarrow \bar{\nu}_{n}(X)=\nu_{n}\left(X \cap A_{n}\right)\right) .
$$

Note that (see 13], Lemma 1.1, p. 354) for an arbitrary Borel set $X \subseteq \mathbf{R}^{N}$ there exists a limit

$$
\nu_{\Delta}(X)=\lim _{n \rightarrow \infty} \bar{\nu}_{n}(X)
$$

Moreover, the functional $\nu_{\Delta}$ is a nontrivial $\sigma$-finite measure defined on the Borel $\sigma$-algebra $B\left(\mathbf{R}^{N}\right)$.

Recall that an element $h \in \mathbf{R}^{N}$ is called an admissible translation in the sense of invariance for the measure $\nu_{\Delta}$ if

$$
(\forall X)\left(X \in B\left(\mathbf{R}^{N}\right) \rightarrow \nu_{\Delta}(X+h)=\nu_{\Delta}(X)\right) .
$$

We define

$$
G_{\Delta}=\left\{h: h \in \mathbf{R}^{N} \& h \text { is an admissible translation for } \nu_{\Delta}\right\} .
$$

It is easy to show that $G_{\Delta}$ is a vector subspace of $\mathbf{R}^{N}$.

We have the following

Lemma 3.1. (13, Theorem 1.4, p.356) The following conditions are equivalent:

$$
\text { 1) } g=\left(g_{1}, g_{2}, \cdots\right) \in G_{\Delta}
$$

2) $\left(\exists n_{g}\right)\left(n_{g} \in N \rightarrow\right.$ the series $\sum_{i \geq n_{g}} \ln \left(1-\frac{\left|g_{i}\right|}{b_{i}-a_{i}}\right)$ is convergent $)$. 
Let $\mathbf{R}^{(N)}$ be the space of all finite sequences, i.e.,

$$
\mathbf{R}^{(N)}=\left\{\left(g_{i}\right)_{i \in N} \mid\left(g_{i}\right)_{i \in N} \in \mathbf{R}^{N} \& \operatorname{card}\left\{i \mid g_{i} \neq 0\right\}<\aleph_{0}\right\}
$$

It is clear that, on the one hand, for an arbitrary compact infinite-dimensional parallelepiped $\Delta=\prod_{k \in N}\left[a_{k}, b_{k}\right]$, we have

$$
\mathbf{R}^{(N)} \subset G_{\Delta} .
$$

On the other hand, $G_{\Delta} \backslash \mathbf{R}^{(N)} \neq \emptyset$ since an element $\left(g_{i}\right)_{i \in N}$ defined by

$$
(\forall i)\left(i \in N \rightarrow g_{i}=\left(1-\exp \left\{-\frac{b_{i}-a_{i}}{2^{i}}\right\} \times\left(b_{i}-a_{i}\right)\right)\right)
$$

belongs to the difference $G_{\Delta} \backslash \mathbf{R}^{(N)}$.

It is easy to show that the vector space $G_{\Delta}$ is everywhere dense in $\mathbf{R}^{N}$ with respect to the Tychonoff topology since $\mathbf{R}^{(N)} \subset G_{\Delta}$.

Below we present an example of the product of an infinite family of Borel probability measures on $R$ with strictly positive continuous distribution functions and a Moore-Yamasaki-Kharazishvili type measure in $\mathbb{R}^{\mathbb{N}}$, such that these measures are equivalent.

Let $\left(c_{n}\right)_{n \in N}$ be a sequence of positive numbers such that $0<c_{n}<l$. On the real axis $R$, for each $n$ consider a continuous function $f_{n}(x)$ which satisfies:

$$
\begin{gathered}
0<f_{n}(x)<1, \int_{-\infty}^{+\infty} f_{n}(x) d x=1, \\
f_{n}(x)=c_{k} \text { for } x \in[0,1] .
\end{gathered}
$$

Such a function $f_{n}(x)$ exists certainly for any $n \in N$.

For $n \in N$, let us denote by $\mu_{n}$ a Borel probability measure on $R$ defined by the distribution density function $f_{n}$.

Fact 3.1 If $\prod_{n \in N} c_{n}>0$, then the measures $\prod_{n \in N} \mu_{n}$ and $\nu_{[0,1]^{N}}$ are equivalent.

Proof. By the Fubini theorem, one can easily prove that the measure $\prod_{n \in N} \mu_{n}$ is $\mathbf{R}^{(N)}$-quasiinvariant. According to 14 , every product measure on $\mathbf{R}^{N}$ is $\mathbf{R}^{(N)}$ ergodic. Therefore, $\prod_{n \in N} \mu_{n}$, hence $\nu_{[0,1]^{N}}$, too is $\mathbf{R}^{(N)}$ - ergodic.

For $x=\left(x_{n}\right) \in \mathbf{R}^{N}$, define a function $f(x)$ by:

$$
f(x)=\prod_{n \in N} f_{n}(x) .
$$

Since $0<f\left(x_{n}\right)<1$, the partial product decreases monotonically, so that the infinite product in (3.1) exists certainly. If $x \in A_{n}$, then $x_{k} \in[0,1]$ for $k>n$, so we have

$$
f(x)=\prod_{k=1}^{n} f_{k}\left(x_{k}\right) \prod_{k>n} c_{k}>0 .
$$

Thus $f(x)$ is positive on $A_{n}$, hence positive on $\cup_{n \in N} A_{n}$, too. On the other hand, since $\nu_{[0,1]^{N}}\left(\mathbf{R}^{N} \backslash \cup_{n \in N} A_{n}\right)=0$, we see that $f(x)$ is positive for $\nu_{[0,1]^{N} \text {-almost all }}$ $x$.

Now, define a measure $\nu^{\prime}$ on $\mathbf{R}^{N}$ by

$$
\nu^{\prime}(X)=\int_{X} f(x) d \nu_{[0,1]^{N}}(x)
$$


for $X \in \mathbf{B}\left(\mathbf{R}^{N}\right)$.

Let us show that $\prod_{n \in N} \mu_{n}=\nu^{\prime}$. For this it suffices to show that for each $A \in \mathbf{B}\left(R^{n}\right)$ we have

$$
\nu^{\prime}\left(A \times \mathbf{R}^{N \backslash\{1, \cdots, n\}}\right)=\prod_{n \in N} \mu_{n}\left(A \times \mathbf{R}^{N \backslash\{1, \cdots, n\}}\right) .
$$

Indeed, we have

$$
\begin{gathered}
\nu^{\prime}\left(A \times \mathbf{R}^{N \backslash\{1, \cdots, n\}}\right)=\int_{A \times \mathbf{R}^{N \backslash\{1, \cdots, n\}}} f(x) d \nu_{[0,1]^{N}}(x)= \\
\lim _{m \rightarrow+\infty} \int_{A_{m} \cap\left(A \times \mathbf{R}^{N \backslash\{1, \cdots, n\}}\right)} f(x) d \nu_{[0,1]^{N}}(x)= \\
\lim _{m \rightarrow+\infty} \int_{A \times \prod_{k=n+1}^{m} \mathbf{R} \times \prod_{k>m}[0,1]} f(x) d \nu_{[0,1]^{N}}(x)= \\
\lim _{m \rightarrow+\infty} \int_{A \times \prod_{k=n+1}^{m} \mathbf{R} \times \prod_{k>m}[0,1]} f(x) d\left(\prod_{k=1}^{m} \mu_{k} \times \prod_{k>m} \lambda_{k}\right)= \\
\lim _{m \rightarrow+\infty} \int_{A \times \prod_{k=n+1}^{m} \mathbf{R}}\left(\int_{\prod_{k>m}[0,1]} f(x) d \prod_{k>m} \lambda_{k}\right) d \prod_{k=1}^{m} \mu_{k}= \\
\lim _{m \rightarrow+\infty} \int_{\prod_{k>m}[0,1]} \prod_{k>m} f_{k}\left(x_{k}\right) d \prod_{k>m} \lambda_{k} \times \\
\lim _{m \rightarrow+\infty} \int_{A \times \prod_{k=n+1}^{m} \mathbf{R}} \prod_{k=1}^{m} f_{k}\left(x_{k}\right) d \prod_{k=1}^{m} \mu_{k}= \\
\lim _{m \rightarrow+\infty} \int_{A} \prod_{k=1}^{n} \int_{\prod_{k}\left(x_{k}\right) d} \prod_{k=1}^{n} \mu_{k} \times \int_{\prod_{k=n+1}^{m} \mathbf{R}} \prod_{k=n+1}^{n} f_{k}\left(x_{k}\right) d \prod_{k=n+1}^{m} \mu_{k}= \\
\prod_{m \rightarrow+\infty} f_{k>m}\left(x_{k}\right) d \prod_{k>m} \lambda_{k} \times \\
c_{k} \times \prod_{k=1}^{n} \mu_{k}(A)=\prod_{k=1} \mu_{k}(A)=\prod_{k \in N} \mu_{k}\left(A \times \mathbf{R}^{N \backslash\{1, \cdots, n\}}\right) .
\end{gathered}
$$

This ends the proof of Fact 3.1.

Remark 3.2. Let the product-measure $\prod_{k \in N} \mu_{k}$ comes from Fact 3.1. Then by virtue of Lemma 3.1, we know that the group of all admissible translations (in the sense of invariance) for the measure $\nu_{[0,1]^{N}}$ is $l_{1}=\left\{\left(x_{k}\right)_{k \in N}:\left(x_{k}\right)_{k \in N} \in\right.$ $\left.R^{N} \& \sum_{k \in N}\left|x_{k}\right|<+\infty\right\}$. Following Fact 3.1, the measures $\prod_{k \in N} \mu_{k}$ and $\nu_{[0,1]^{N}}$ are equivalent, which implies that the group of all admissible translations (in the sense of quasiinvariance) for the measure $\prod_{k \in N} \mu_{k}$ is equal to $l_{1}$.

For $\left(x_{k}\right)_{k \in N} \in l_{1}$, we set $\nu_{k}(X)=\mu_{k}\left(X-x_{k}\right)$ for each $X \in B(R)$. It is obvious that $\mu_{k}$ and $\nu_{k}$ are equivalent for each $k \in N$. For $k \in N$ and $x \in R$, we put $\rho_{k}(x)=\frac{d \nu_{k}(x)}{d \mu_{k}(x)}$. Let us consider the product-measures $\mu=\prod_{k \in N} \mu_{k}$ and $\nu=\prod_{k \in N} \nu_{k}$.

On the one hand, following our observation, the measures $\mu$ and $\nu$ are equivalent. On the other hand, by virtue of Kakutani's well known result (see, [8]), since the 
measures $\mu$ and $\nu$ are equivalent, we deduce that the infinite product $\prod_{k \in N} \alpha_{k}$ is divergent to zero, where $\alpha_{k}=\int_{R} \sqrt{\rho_{k}\left(x_{k}\right)} d \mu_{k}\left(x_{k}\right)$. In this case $r_{n}(x)=\prod_{k=1}^{n} \rho_{k}(x)$ is convergent (in the mean) to the function $r(x)=\prod_{k=1}^{\infty} \rho_{k}(x)$ which is the density of the measure $\nu$ with respect to $\mu$, i.e.,

$$
r(x)=\frac{d \nu(x)}{d \mu(x)} .
$$

Remark 3.3. The approach used in the proof of Fact 3.1 is taken from [19] (see Proposition 4.1, p. 702).

In the context of Fact 3.1 we state the following

Problem 3.1 Do there exist a family $\left(\mu_{k}\right)_{k \in N}$ of linear Gaussian probability measures on $R$ and a Moore-Yamasaki-Kharazishvili type measure $\lambda$ in $\mathbf{R}^{\mathbf{N}}$ such that the measures $\prod_{k \in N} \mu_{k}$ and $\lambda$ are equivalent?

\section{Solution of Problems $1.3-1.4$}

We present a new approach for the solution of Problems 1.3 - 1.4, which is quite different from the approach introduced in [8]. Our approach uses the technique of the so-called uniformly distributed sequences. The main notions and auxiliary propositions are taken from [10].

Definition 4.1. [10] A sequence $\left(x_{k}\right)_{k \in N}$ of real numbers from the interval $(a, b)$ is said to be equidistributed or uniformly distributed on an interval $(a, b)$ if for any subinterval $[c, d]$ of $(a, b)$ we have

$$
\lim _{n \rightarrow \infty} n^{-1} \#\left(\left\{x_{1}, x_{2}, \cdots, x_{n}\right\} \cap[c, d]\right)=(b-a)^{-1}(d-c),
$$

where \# denotes the counting measure.

Now let $X$ be a compact Polish space and $\mu$ be a probability Borel measure on $X$. Let $\mathcal{R}(X)$ be a space of all bounded continuous measurable functions defined on $X$.

Definition 4.2. A sequence $\left(x_{k}\right)_{k \in N}$ of elements of $X$ is said to be $\mu$-equidistributed or $\mu$-uniformly distributed on $X$ if for every $f \in \mathcal{R}(X)$ we have

$$
\lim _{n \rightarrow \infty} n^{-1} \sum_{k=1}^{n} f\left(x_{k}\right)=\int_{X} f d \mu .
$$

Definition 4.3. ([10], Lemma 2.1, p. 199) Let $f \in \mathcal{R}(X)$. Then, for $\mu^{N}$-almost every sequence $\left(x_{k}\right)_{k \in N} \in X^{N}$, we have

$$
\lim _{n \rightarrow \infty} n^{-1} \sum_{k=1}^{n} f\left(x_{k}\right)=\int_{X} f d \mu .
$$

Lemma 4.4. (10, pp. 199-201) Let $S$ be a set of all $\mu$-equidistributed sequences on $X$. Then we have $\mu^{N}(S)=1$.

Corollary 4.5. ([20], Corollary 2.3, p. 473) Let $\ell_{1}$ be a Lebesgue measure on $(0,1)$. Let $D$ be a set of all $\ell_{1}$-equidistributed sequences on $(0,1)$. Then we have $\ell_{1}^{N}(D)=1$. 
Definition 4.6. Let $\mu$ be a probability Borel measure on $R$ with a distribution function $F$. A sequence $\left(x_{k}\right)_{k \in N}$ of elements of $R$ is said to be $\mu$-equidistributed or $\mu$-uniformly distributed on $R$ if for every interval $[a, b](-\infty \leq a<b \leq+\infty)$ we have

$$
\lim _{n \rightarrow \infty} n^{-1} \#\left([a, b] \cap\left\{x_{1}, \cdots, x_{n}\right\}\right)=F(b)-F(a) .
$$

Lemma 4.7. (20, Lemma 2.4, p. 473) Let $\left(x_{k}\right)_{k \in N}$ be an $\ell_{1}$-equidistributed sequence on $(0,1), F$ be a strictly increasing continuous distribution function on $R$ and $p$ be a Borel probability measure on $R$ defined by $F$. Then $\left(F^{-1}\left(x_{k}\right)\right)_{k \in N}$ is p-equidistributed on $R$.

Corollary 4.8. (20], Corollary 2.4, p. 473) Let F be a strictly increasing continuous distribution function on $R$ and $p$ be a Borel probability measure on $R$ defined by $F$. Then for a set $D_{F} \subset R^{N}$ of all p-equidistributed sequences on $R$ we have :

(i) $D_{F}=\left\{\left(F^{-1}\left(x_{k}\right)\right)_{k \in N}:\left(x_{k}\right)_{k \in N} \in D\right\}$;

(ii) $p^{N}\left(D_{F}\right)=1$.

Lemma 4.9. Let $F_{1}$ and $F_{2}$ be different strictly increasing continuous distribution functions on $R$, and $p_{1}$ and $p_{2}$ be Borel probability measures on $R$ defined by $F_{1}$ and $F_{2}$, respectively. Then there does not exist a sequence of real numbers $\left(x_{k}\right)_{k \in \mathbf{N}}$ which simultaneously is $p_{1}$-equidistributed and $p_{2}$-equidistributed.

Proof. Assume the contrary and let $\left(x_{k}\right)_{k \in \mathbf{N}}$ be such a sequence. Since $F_{1}$ and $F_{2}$ are different, there is a point $x_{0} \in \mathbb{R}$ such that $F_{1}\left(x_{0}\right) \neq F_{2}\left(x_{0}\right)$. The latter relation is not possible under our assumption because $\left(x_{k}\right)_{k \in \mathbf{N}}$ simultaneously is $p_{1}$-equidistributed and $p_{2}$-equidistributed, which implies

$$
F_{1}\left(x_{0}\right)=\lim _{n \rightarrow \infty} n^{-1} \#\left(\left(-\infty, x_{0}\right] \cap\left\{x_{1}, \cdots, x_{n}\right\}\right)=F_{2}\left(x_{0}\right) .
$$

The next theorem contains the solution of Problem 1.3.

Theorem 4.10. Let $F_{1}$ and $F_{2}$ be different strictly increasing continuous distribution functions on $R$ and $p_{1}$ and $p_{2}$ be Borel probability measures on $R$, defined by $F_{1}$ and $F_{2}$, respectively. Then the measures $p_{1}^{N}$ and $p_{2}^{N}$ are orthogonal.

Proof. Let $D_{F_{1}}$ and $D_{F_{2}}$ denote $p_{1}$-equidistributed and $p_{2}$-equidistributed sequences on $R$, respectively. By Lemma 4.9 we know that $D_{F_{1}} \cap D_{F_{2}}=\emptyset$. By Corollary 4.8 we know that $p_{1}^{N}\left(D_{F_{1}}\right)=1$ and $p_{2}^{N}\left(D_{F_{2}}\right)=1$. This ends the proof of the theorem.

Definition 4.11. Let $\left\{\mu_{i}: i \in I\right\}$ be a family of probability measures defined on a measure space $(X, M)$. Let $S(X)$ be defined by

$$
S(X)=\cap_{i \in I} \operatorname{dom}\left(\bar{\mu}_{i}\right)
$$

where $\bar{\mu}_{i}$ denotes a usual completion of the measure $\mu_{i}$. We say that the family $\left\{\mu_{i}: i \in I\right\}$ is strongly separable if there exists a partition $\left\{C_{i}: i \in I\right\}$ of the space $X$ into elements of the $\sigma$-algebra $S(X)$ such that $\bar{\mu}_{i}\left(C_{i}\right)=1$ for each $i \in I$.

Definition 4.12. Let $\left\{\mu_{i}: i \in I\right\}$ be a family of probability measures defined on a measure space $(X, M)$. Let $S(I)$ denote a minimal $\sigma$-algebra generated by singletons of $I$ and the $\sigma$-algebra $S(X)$ of subsets of $X$ be defined by

$$
S(X)=\cap_{i \in I} \operatorname{dom}\left(\bar{\mu}_{i}\right),
$$


where $\bar{\mu}_{i}$ denotes a usual completion of the measure $\mu_{i}$ for $i \in I$. We say that a $(S(X), S(I))$-measurable mapping $T: X \rightarrow I$ is a well-founded estimate of an unknown parameter $i(i \in I)$ for the family $\left\{\mu_{i}: i \in I\right\}$ if the following condition

$$
(\forall i)\left(i \in I \rightarrow \mu_{i}\left(T^{-1}(\{i\})=1\right)\right)
$$

holds true.

One can easily get the validity of the following assertion.

Lemma 4.13. (20], Lemma 2.5, p. 474) Let $\left\{\mu_{i}: i \in I\right\}$ be a family of probability measures defined on a measure space $(X, M)$. The following propositions are equivalent:

(i) The family of probability measures $\left\{\mu_{i}: i \in I\right\}$ is strongly separable;

(ii) There exists a well-founded estimate of an unknown parameter $i(i \in I)$ for the family $\left\{\mu_{i}: i \in I\right\}$.

The next theorem contains the solution of Problem 1.4.

Theorem 4.14. Let $\mathcal{F}$ be a family of all strictly increasing and continuous distribution functions on $\mathbf{R}$ and $p_{F}$ be a Borel probability measure on $R$ defined by $F$ for each $F \in \mathcal{F}$. Then the family of Borel probability measures $\left.\left\{p_{F}^{N}: F \in \mathcal{F}\right)\right\}$ is strongly separable.

Proof. We denote by $D_{F}$ the set of all $p_{F}$-equidistributed sequences on $R$ for each $F \in \mathcal{F}$. By Lemma 4.9 we know that $D_{F_{1}} \cap D_{F_{2}}=\emptyset$ for each different $F_{1}, F_{2} \in \mathcal{F}$. By Corollary 4.8 we know that $p_{F}^{N}\left(D_{F}\right)=1$ for each $F \in \mathcal{F}$. Let us fix $F_{0} \in \mathcal{F}$ and define a family $\left(C_{F}\right)_{F \in \mathcal{F}}$ of subsets of $\mathbf{R}^{\mathbf{N}}$ as follows: $C_{F}=D_{F}$ for $F \in \mathcal{F} \backslash\left\{F_{0}\right\}$ and $C_{F_{0}}=R^{N} \backslash \cup_{F \in \mathcal{F} \backslash\left\{F_{0}\right\}} D_{F}$. Since $D_{F}$ is a Borel subset of $\mathbf{R}^{\mathbf{N}}$ for each $F \in \mathcal{F}$, we claim that $C_{F} \in S\left(\mathbf{R}^{\mathbf{N}}\right)$ for each $F \in \mathcal{F} \backslash\left\{F_{0}\right\}$. Since $\overline{p_{F}^{N}}\left(R^{N} \backslash \cup_{F \in \mathcal{F}} D_{F}\right)=0$ for each $F \in \mathcal{F}$, we deduce that $R^{N} \backslash \cup_{F \in \mathcal{F}} D_{F} \in \cap_{F \in \mathcal{F}} \operatorname{dom}\left(\overline{p_{F}^{N}}\right)=S\left(R^{N}\right)$. Since $S\left(\mathbf{R}^{\mathbf{N}}\right)$ is an $\sigma$-algebra, we claim that $C_{F_{0}} \in S\left(R^{N}\right)$ because $\overline{p_{F}^{N}}\left(R^{N} \backslash \cup_{F \in \mathcal{F}} D_{F}\right)=0$ for each $F \in \mathcal{F}$ (equivalently, $R^{N} \backslash \cup_{F \in \mathcal{F}} D_{F} \in S\left(R^{N}\right)$ ), and

$$
C_{F_{0}}=R^{N} \backslash \cup_{F \in \mathcal{F} \backslash\left\{F_{0}\right\}} D_{F}=\left(R^{N} \backslash \cup_{F \in \mathcal{F}} D_{F}\right) \cup D_{F_{0}} .
$$

This ends the proof of the theorem.

By virtue of the results of Lemma 4.13 and Theorem 4.14 we get the following

Corollary 4.15. Let $\mathcal{F}$ be a family of all strictly increasing and continuous distribution functions on $\mathbf{R}$. Then there exists a well-founded estimate of an unknown distribution function $F(F \in \mathcal{F})$ for the family of Borel probability measures $\left\{p_{F}^{N}: F \in \mathcal{F}\right\}$.

Remark 4.16. The validity of Theorem 4.14 and Corollary 4.15 can be obtained for an arbitrary family of strictly increasing and continuous distribution functions on R. Note that Corollary 4.15 extends the main result established in [20] (see Lemma 2.6, p. 476).

Remark 4.17. The requirements in Theorem 4.14 that all Borel probability measures on $\mathbf{R}$ are defined by strictly increasing and continuous distribution functions on $\mathbf{R}$ and the measures under consideration are infinite powers of the corresponding measures are essential. Indeed, let $\mu$ be a linear Gaussian measure on $\mathbf{R}$ whose 
density distribution function has the form $f(x)=\frac{1}{\sqrt{2 \pi}} e^{-\frac{x^{2}}{2}}(x \in \mathbf{R})$. Let $\delta_{x}$ be a Dirac measure defined on the Borel $\sigma$-algebra of subsets of $\mathbf{R}$ and concentrated at $x(x \in \mathbf{R})$. Let $D$ be a subset of $\mathbf{R}^{\mathbf{N}}$ defined by

$$
D=\left\{\left(x_{k}\right)_{k \in N}: \lim _{n \rightarrow \infty} \frac{\sum_{k=1}^{n} x_{k}}{n}=0\right\} .
$$

It is obvious that $D$ is a Borel subset of $\mathbf{R}^{\mathbf{N}}$.

For $\left(x_{k}\right)_{k \in N} \in D$ we set $\mu_{\left(x_{k}\right)_{k \in N}}=\prod_{k \in N} \delta_{x_{k}}$ 1]. Let us consider the family of Borel probability measures $\left\{\mu^{N}\right\} \cup\left\{\mu_{\left(x_{k}\right)_{k \in N}}:\left(x_{k}\right)_{k \in N} \in D\right\}$. It is obvious that it is an orthogonal family of Borel product-measures for which Theorem 4.14 fails. Indeed, assume the contrary and let $\{C\} \cup\left\{C_{\left(x_{k}\right)_{k \in N}}:\left(x_{k}\right)_{k \in N} \in D\right\}$ be such a partition of $\mathbf{R}^{\mathbf{N}}$ into elements of the $\sigma$-algebra $S_{0}\left(R^{N}\right)=\cap_{\left(x_{k}\right)_{k \in N} \in D} \operatorname{dom}\left(\bar{\mu}_{\left(x_{k}\right)_{k \in N}}\right) \cap$ $\operatorname{dom}\left(\overline{\mu^{N}}\right)$ that $\bar{\mu}_{\left(x_{k}\right)_{k \in N}}\left(C_{\left(x_{k}\right)_{k \in N}}\right)=1$ for $\left(x_{k}\right)_{k \in N} \in D$ and $\overline{\mu^{N}}(C)=1$. Since $\left(x_{k}\right)_{k \in N} \in C_{\left(x_{k}\right)_{k \in N}}$ for each $\left(x_{k}\right)_{k \in N} \in D$ we deduce that $D \cap C=\emptyset$. This implies that $\overline{\mu^{N}}(C) \leq \overline{\mu^{N}}\left(\mathbf{R}^{\mathbf{N}} \backslash D\right)=0$ because by the strong law of large numbers we have that $\overline{\mu^{N}}(D)=1$. The latter relation is a contradiction and Remark 4.17 is proved.

\section{REFERENCES}

[1] Cameron R.H., Martin W.T. On Transformations of Wiener integrals under translations // Ann.of Math.-1944.- 45.- P. 386-396.

[2] Feldman J. Equivalence and orthogonality of Gaussian processes // Pacific J. Math. - 1958.-8.P. 699-708.

[3] Gill T., Kirtadze A., Pantsulaia G., Plichko A. Existence and uniqueness of translation invariant measures in separable Banach spaces // Funct. Approx. Comment. Math.-2014.50(2).-P. 401-419.

[4] Gill T.L., Pantsulaia G.R., and Zachary W.W. Constructive Analysis In Infinitely Many Variables // Communications in Mathematical Analysis.-2012.-13 (1).-P. 107-141.

[5] Girsanov I.V., Mityasin B.S. Quasi-invariant measures and linear topological spaces (in Russian)// Nauchn. Dokl. Vys. Skol.-1959.-2.-P. 5-10.

[6] Grenander Ulf. Stochastic processes and statistical inference // Ark. Mat.-1950.- 1.-P. 195277.

[7] Hill D. G. B. $\sigma$-finite invariant measures on infinite product spaces // Trans. Amer. Math. Soc.-1971.- 153.- P. 347-370.

[8] Kakutani S. On equivalence of infinite product measures // Ann. Math.- 1948.- 4 (9).- P. 214-224.

[9] Kharazishvili A.B. On invariant measures in the Hilbert space (in Russian) // Bull. Acad. Sci.Georgian SSR.-1984.- 114(1).-P. 41-48.

[10] Kuipers L., Niederreiter H. Uniform distribution of sequences.-New York etc.: John Wiley \& Sons, 1974.

[11] LePage R.D., Mandrekar V. Equivalence-singularity dichotomies from zero-one laws // Proc. Amer. Math. Soc.-1972.- 31.-P. 251-254.

[12] Moore C.C. Invariant measures on product spaces // Proc. Fifth Berkeley Sympos. Math. Statist. and Probability.-1965-1966.- Vol. II: Contributions to Probability Theory, Part 2.- P. $447-459$

[13] Pantsulaia G. Duality of measure and category in infinite-dimensional separable Hilbert space $l_{2}$ // Int. J. Math. Math. Sci.-2002.-30(6).-P. 353-363. MR 1904675

[14] Shimomura, Hiroaki. An aspect of quasi-invariant measures on $R^{\infty} / /$ Publ. Res. Inst. Math. Sci.-1975/76.- 11(3).- P. 749-773.

[15] Skorokhod A.V. Integration in Hilbert space (in Russian).- Moscow, 1974. English transl.: Springer, 1975.

[16] Sudakov V.N.Linear sets with quasi-invariant measure(in Russian)// Dokl. Akad. Nauk SSSR.-1959.- 127.-P. 524-525.

${ }^{1}$ Note that $\prod_{k \in N} \delta_{x_{k}}=\delta_{\left(x_{k}\right)_{k \in N}}$. 
[17] Veršik A. M., Duality in the theory of measure in linear spaces (in Russian) //Dokl. Akad. Nauk SSSR.-1966.- 170.-P. 497-500.

[18] Xia D.X. Measure and integration on infinite-dimensional spaces.-New York.: Academic Press, 1972.

[19] Yamasaki Y. Translationally invariant measure on the infinite-dimensional vector space // Publ. Res. Inst. Math. Sci.-1980. - 16(3).-P. 693-720.

[20] Zerakidze Z., Pantsulaia G., Saatashvili G. On the separation problem for a family of Borel and Baire $G$-powers of shift-measures on $\mathbb{R} / /$ Ukrainian Math. J. -2013.- 65 (4).- P. 470-485.

Department of Mathematics, Georgian Technical University, Kostava Street-77, Tbilisi 0175, Georgia

E-mail address: m.kintsurashvili@gtu.ge, t.kiria@gtu.ge, g.pantsulaia@gtu.ge 